\title{
Lagrange-Galerkin methods for the incompressible Navier-Stokes equations: a review
}

\author{
Rodolfo Bermejo $^{1 *}$, Laura Saavedra ${ }^{2,1}$ \\ ${ }^{1}$ Departamento de Matemática Aplicada a la Ingeniería Industrial ETSII , \\ Universidad Politecnica de Madrid, Spain \\ ${ }^{2}$ Departamento de Matemática Aplicada a la Ingeniería Aeroespacial ETSAA, \\ Universidad Politecnica de Madrid, Spain \\ *Email address for correspondence: rbermejo@etsii.upm.es \\ Communicated by Roberto Ferretti \\ Received on May 10, 2015. Accepted on November 9, 2015.
}

\begin{abstract}
We review in this paper the development of Lagrange-Galerkin (LG) methods to integrate the incompressible Navier-Stokes equations (NSEs) for engineering applications. These methods were introduced in the computational fluid dynamics community in the early eighties of the past century, and at that time they were considered good methods for both their theoretical stability properties and the way of dealing with the nonlinear terms of the equations; however, the numerical experience gained with the application of LG methods to different problems has identified drawbacks of them, such as the calculation of specific integrals that arise in their formulation and the calculation of the flow trajectories, which somehow have hampered the applicability of LG methods. In this paper, we focus on these issues and summarize the convergence results of LG methods; furthermore, we shall briefly introduce a new stabilized LG method suitable for high Reynolds numbers.
\end{abstract}

Keywords: Lagrange-Galerkin, finite elements, Navier-Stokes.

AMS subject classification: 65M12, 65M25, 65M60.

\section{Introduction}

LG methods, also known as Semi-Lagrangian (SL) methods in the Numerical Wether Prediction community, are efficient numerical techniques to integrate time dependent convection-diffusion problems, including the incompressible NSEs. The distinctive feature of both LG and SL methods is the way they deal with the material derivative. They discretize the material derivative backward in time along flow trajectories, but LG methods calculate the quantities at the feet of the trajectories by a Galerkin projection onto a suitable finite dimensional space, generally a finite element space, whereas SL methods use polynomial interpolation of order higher than one; so, LG can be applied with any type of mesh in contrast with SL 


\section{R. Bermejo, L. Saavedra}

methods that are mostly applied with structured quadrilateral meshes. In this paper, we review the development of LG methods to integrate NSEs in an engineering context in which the use of unstructured meshes is almost mandatory. LG methods were introduced in [10] and [23]. The application of LG methods to integrate NSEs has some advantages, such as numerical stability and the way of dealing with the nonlinear terms. It is known that in the integration of NSEs by conventional implicit time marching schemes, the nonlinear terms yield an algebraic system of nonlinear equations that is solved by an iterative procedure, increasing thus the number of arithmetic operations to reach the solution and requiring a large space of memory. In contrast, backward integration of the material derivative along trajectories, which is a natural way of introducing upwinding in the space discretization of the equations, transforms the NSEs into a linear Stokes problem, so at each time step one has to solve an algebraic linear system of equations that is more manageable than the algebraic nonlinear system of equations produced by conventional implicit time marching schemes; furthermore, we must remark that upwinding along the trajectories is a numerical mechanism to stabilize the convective terms. A priori, these assets make LG methods look like efficient methods to integrate NSEs ; however, they have a drawback concerned with the calculation of the integrals of the form $\int_{K} \phi_{j}\left(X_{h}\left(x, t_{n+1}, t_{n}\right)\right) \phi_{i}(x) d x$, which appear in the formulation of the numerical solution, here $K$ is a generic element, $\phi_{i}$ is the ith global basis function of the finite element space and $X_{h}\left(x, t_{n+1}, t_{n}\right)$ is the foot of the characteristic associated with the point $x$. For stability and optimal convergence reasons these integrals have to be calculated with high accuracy, see [20] and [6], thus requiring the use of high order quadrature rules. Since each quadrature point has an associated foot of characteristic, this means that many systems of differential equations have to be solved backward in time by a numerical method incorporating a point searching algorithm to identify the element of the mesh where each foot is located. The location of points inside the elements of a mesh is a trivial task in structured meshes, for instance, in meshes composed of squares or hexahedra, but if the mesh is unstructured the location of points is not that simple; hence, LG methods may become less efficient than they look at first. To partially overcome these drawbacks, some variations of conventional LG method, such as the area-weighting method for quadrilateral structured meshes [20], exact integration [22] for straight side triangular meshes with linear elements, and the modified LG methods $[5,6]$, have been proposed. We do not consider such variations in this paper.

We introduce some notation about the functional spaces we use in the paper. For $s \geq 0$ real and real $1 \leq p \leq \infty, W^{s, p}(\Omega)$ denotes the real Sobolev 


\section{LG methods for Navier-Stokes Equations}

spaces defined on $\Omega \subset \mathbb{R}^{d}$ for scalar real-valued functions. $\|\cdot\|_{W^{s, p}(\Omega)}$ and $|\cdot|_{W^{s, p}(\Omega)}$ denote the norm and semi-norm, respectively, of $W^{s, p}(\Omega)$. When $s=0, W^{0, p}(\Omega):=L^{p}(\Omega)$. For $p=2$, the spaces $W^{s, 2}(\Omega)$ are denoted by $H^{s}(\Omega)$, which are real Hilbert spaces with inner product $(\cdot, \cdot)_{s}$. For $s=0$, $H^{0}(\Omega):=L^{2}(\Omega)$, the inner product in $L^{2}(\Omega)$ is denoted by $(\cdot, \cdot) . H_{0}^{1}(\Omega)$ is the space of functions of $H^{1}(\Omega)$ which vanish on the boundary $\partial \Omega$ in the sense of trace. $H^{-1}$ denotes the dual of $H_{0}^{1}(\Omega)$. The corresponding spaces of real vector (and tensor)-valued functions, $v: \Omega \rightarrow \mathbb{R}^{d}$ are denoted by boldface letters; for instance, $\mathbf{W}^{s, p}(\Omega):=\left(W^{s, p}(\Omega)\right)^{d}:=\left\{v: \Omega \rightarrow \mathbb{R}^{d}\right.$ : $\left.v_{i} \in W^{s, p}(\Omega), 1 \leq i \leq d\right\}$. Let $X$ be a real Banach space $\left(X,\|\cdot\|_{X}\right)$, if $v:(0, T) \rightarrow X$ is a strongly measurable function with values in $X$, we denote by $L^{p}(X), H^{s}(X)$ and $C(X)$ the spaces $L^{p}(0, T ; X), H^{s}(0, T ; X)$ and $C([0, T] ; X)$ respectively. $C^{r, 1}(\bar{\Omega}), r \geq 0$, is the space of functions defined in the closure of $\Omega, r$ times differentiable and with the $r$ th derivative being Lipschitz continuous.

The layout of the paper is as follows. In Section 2, we introduce the semidiscrete Lagrange formulation of the incompressible NSEs to motivate the formulation of LG methods. In Section 3, a detailed description of first and second order in time conventional LG methods is presented, paying attention to important issues such as the efficient implementation of the methods and the calculation of the feet of the characteristic curves. Section 4 is devoted to the presentation of the error analysis, whereas in Section 5 we illustrate the performance of conventional LG-BDF methods in some three dimensional benchmark problems. In Section 6, we present the implementation of LG methods in the framework of projection methods. Finally, some new developments of LG methods for high Reynolds numbers are introduced in Section 7.

Throughout this paper, $C$ will denote a generic positive constant which is independent of $h$ and $\Delta t$. $C$ will have different values at different places of appearance.

\section{Semidiscrete Lagrangian formulations of Navier-Stokes equa- tions}

Let $\Omega \subset \mathbb{R}^{d}(d=2$ or 3 ) be a bounded domain with Lipschitz boundary $\Gamma$ and let $[0, T]$ denote a time interval. We consider the following Cauchy problem:

Find the functions $v: \Omega \times(0, T] \rightarrow \mathbb{R}^{d}$ and $p: \Omega \times(0, T] \rightarrow \mathbb{R}$ that 


\section{R. Bermejo, L. Saavedra}

satisfy

$$
\left\{\begin{aligned}
\frac{\partial v}{\partial t}+(v \cdot \nabla) v-\nu \Delta v+\nabla p=f, & \text { in } \Omega \times(0, T), \\
\operatorname{div} v=0, & \text { in } \Omega \times(0, T),
\end{aligned}\right.
$$

and the following initial and boundary conditions

$$
\begin{aligned}
v(\cdot, 0) & =v^{0}(\cdot) \text { in } \Omega, \\
v & =0 \text { on } \Gamma \times(0, T) .
\end{aligned}
$$

Physically, system (1) describes the unsteady flow of a constant density Newtonian fluid with constant kinematic viscosity $\nu>0 . v, p$, and $f: \bar{\Omega} \times$ $(0, T) \rightarrow \mathbb{R}^{d}$ denote the flow velocity, the pressure and the density of body forces per unit of mass, respectively. A divergence free velocity $v^{0}: \Omega \rightarrow \mathbb{R}^{d}$ is prescribed at the initial instant $t=0$. Recalling the expression of the material derivative, $\frac{D v}{D t}:=\frac{\partial v}{\partial t}+v \cdot \nabla v$, we calculate a numerical solution using the following weak formulation:

Given $f \in L^{2}\left(\mathbf{H}^{-1}\right)$ and $v^{0} \in \mathbf{H}$, find $v \in L^{2}\left(\mathbf{H}_{0}^{1}(\Omega)\right) \cap L^{\infty}\left(\mathbf{L}^{2}(\Omega)\right)$ and $p \in L^{2}\left(L_{0}^{2}(\Omega)\right)$, such that for all $u \in \mathbf{H}_{0}^{1}(\Omega)$ and $q \in L_{0}^{2}(\Omega)$ :

$$
\left\{\begin{array}{l}
\left(\frac{D v}{D t}, u\right)+\nu(\nabla v, \nabla u)-(p, \operatorname{div} u)=(f, u), \\
(\operatorname{div} v, q)=0
\end{array}\right.
$$

where $\mathbf{H}:=\left\{v \in \mathbf{L}^{2}(\Omega): \operatorname{div} v=0\right.$ and $\left.\left.\mathbf{n} \cdot v\right|_{\Gamma}=0\right\}, \mathbf{H}^{-1}$ is the dual space of $\mathbf{H}_{0}^{1}(\Omega), L_{0}^{2}(\Omega):=\left\{q \in L^{2}(\Omega): \int_{\Omega} q d x=0\right\}$, and $\mathbf{n}$ is the unit outward normal vector.

In this paper, we focus on the approximation of equations (1)-(3) based on the backward in time discretization along the characteristic curves $X(x, s ; t)$ of the operator $\frac{D}{D t}=\frac{d}{d t}+v \cdot \nabla$, which are solution of the initial value problem

$$
\frac{d X(x, s ; t)}{d t}=v(X(x, s ; t), t), \quad X(x, s ; s)=x .
$$

$t \rightarrow X(x, s ; t)$ can be viewed as the trajectory of a fluid particle that at time $s$ is at the point $x$. It is well known that if $v \in C^{0}\left(\mathbf{C}^{0,1}(\bar{\Omega})\right)$, the solution $X(x, s ; t)$ is unique and can be represented by the integral form

$$
X(x, s ; t)=x+\int_{s}^{t} v(X(x, s ; \tau), \tau) d \tau .
$$




\section{LG methods for Navier-Stokes Equations}

The mapping $x \rightarrow X(x, s ; t)$ has the group property; i.e., let $t_{1}$ and $t_{2} \in$ $[0, T]$, then $X\left(x, s ; t_{2}\right)=X\left(\cdot, t_{1} ; t_{2}\right) \circ X\left(x, s ; t_{1}\right)$. Hereafter, unless otherwise stated, we adopt the notation $X^{k, l}(x):=X\left(x, t_{l} ; t_{k}\right), k$ and $l$ being positive integers. The following results are well known.

Lemma 2.1. Assume that $v \in C^{0}\left(\mathbf{C}^{0,1}(\bar{\Omega})\right)$ and $s-\tau$ is sufficiently small, then $x \rightarrow X(x, s ; t)$ is a quasi-isometric homeomorphism of $\Omega$ onto $\Omega$ and its Jacobian determinant $J=1$ a.e. in $\Omega$. Moreover,

$$
K_{1}|x-y| \leq|X(x, s ; t)-X(y, s ; t)| \leq K_{2}|x-y|,
$$

where $K_{2}=\exp \left(|s-t| \cdot|\nabla \mathbf{u}|_{L^{\infty}\left(\mathbf{L}^{\infty}(D)^{d}\right)}\right.$ and $K_{1}=(1-|s-t| \cdot \mid$ $\left.\left.\nabla \mathbf{u}\right|_{L^{\infty}\left(\mathbf{L}^{\infty}(D)^{d}\right)} K_{2}\right)$, and $|a-b|$ denotes the Euclidean distance between the points $a$ and $b \in \mathbb{R}^{d}$.

For a proof of this lemma see [27]. In the following lemma we collect some facts concerning the solution to (5) which are standard in the theory of ODE systems.

Lemma 2.2. Assume that $v \in C^{0}\left(\mathbf{C}^{k-1,1}(\bar{\Omega})\right), k \geq 1$. Then for any integer $n, 0 \leq n \leq N-1$, the unique solution $t \rightarrow X\left(x, t_{n+1} ; t\right)$, $\left(t \in\left[t_{n}, t_{n+1}\right] \subset[0, T]\right)$ to (5) is such that $X\left(x, t_{n+1} ; t\right) \in C^{0,1}\left(\mathbf{C}^{k-1,1}(\overline{\Omega)})\right.$. Furthermore, let the multi-index $\alpha \in \mathbb{N}^{d}$, then for all $\alpha, 1 \leq|\alpha| \leq k$, it follows that $\left.\partial_{x_{j}}^{|\alpha|} X_{i}\left(x, t_{n+1} ; t\right) \in C\left([0, T] ; \mathbf{L}^{\infty}(\Omega) \times[0, T]\right)\right), 1 \leq i, j \leq d$.

To motivate the introduction of LG methods, we shall derive the Lagrangian formulation of the NSEs. Let $s, t \in[0, T]$ and $x=X(y, s ; t), x, y \in$ $\Omega$, then it follows that $\frac{d X}{d t}=v(x, t)$. We assume that $y \rightarrow X(y, s ; t)$ is a diffeomorphism with Jacobian matrix $\mathbf{F}(y, s ; t):=\left(\frac{\partial X(y, s ; t)}{\partial y}\right)$ the determinant of which is denoted by $J(y, s ; t)$. Setting $c(x, t)=c(X(y, s ; t), t)=$ $\bar{c}(y, t)$, where $c$ is either a scalar- or a vector-valued function, we can easily compute

$$
\begin{aligned}
& \frac{\partial \bar{c}}{\partial t}=\frac{\partial c}{\partial t}+v \cdot \nabla c=\frac{D c}{D t}, \quad \nabla c=\mathbf{F}^{-T} \nabla \bar{c}, \\
& \nu \Delta c=\nu \operatorname{div}(\nabla c)=\frac{\nu}{J} \operatorname{div}\left(J \mathbf{F}^{-1} \mathbf{I F}^{-T} \nabla \bar{c}\right),
\end{aligned}
$$

where $\mathbf{I}$ is the unit matrix and $\mathbf{F}^{-T}$ is the transpose of $\mathbf{F}^{-1}$. Let $I_{n}:=$ 


\section{R. Bermejo, L. Saavedra}

$\left[t_{n}, t_{n+1}\right]$, then the NSEs can be recast in $\Omega \times I_{n}$ as

$$
\left\{\begin{array}{l}
\frac{\partial \bar{v}}{\partial t}+\mathbf{F}^{-T} \nabla \bar{p}-\frac{\nu}{J} \operatorname{div}\left(J \mathbf{F}^{-1} \mathbf{I F}^{-T} \nabla \bar{v}\right)-\bar{f}=0 \\
\frac{1}{J} \operatorname{div}\left(J \mathbf{F}^{-1} \bar{v}\right)=0 \\
\left.\bar{v}\right|_{\Gamma}=0 \\
\bar{v}\left(y, t_{n}\right) \text { known. }
\end{array}\right.
$$

Letting

$$
\bar{G}(y, t)=-\mathbf{F}^{-T} \nabla \bar{p}+\frac{\nu}{J} \operatorname{div}\left(J \mathbf{F}^{-1} \mathbf{I F}^{-T} \nabla \bar{v}\right)+\bar{f},
$$

and taking as initial time $s=t_{n+1}$, the application of the Backward Differentiation Formula of order 1 (BDF1) to discretize in time (7) yields the semidiscrete system

$$
\left\{\begin{array}{l}
\frac{\bar{v}\left(y, t_{n+1}\right)-\bar{v}\left(y, t_{n}\right)}{\Delta t}=\bar{G}\left(y, t_{n+1}\right)+O(\Delta t) \\
\frac{1}{J} \operatorname{div}\left(J F^{-1} \bar{v}\left(y, t_{n+1}\right)\right)=0 \\
\left.\bar{v}\left(y, t_{n+1}\right)\right|_{\Gamma}=0
\end{array}\right.
$$

Noting that for $t=t_{n+1}, \bar{v}\left(y, t_{n+1}\right)=v\left(x, t_{n+1}\right), \mathbf{F}\left(y, t_{n+1} ; t_{n+1}\right)=\mathbf{I}$, $J\left(y, t_{n+1} ; t_{n+1}\right)=1$, and

$$
\bar{G}\left(y, t_{n+1}\right)=-\nabla p\left(x, t_{n+1}\right)+\nu \Delta v\left(x, t_{n+1}\right)+f\left(x, t_{n+1}\right),
$$

and recalling the notation $X^{n, n+1}(x)=X\left(x, t_{n+1} ; t_{n}\right)$ so that $\bar{v}\left(y, t_{n}\right)=$ $v\left(X^{n, n+1}(x), t_{n}\right)=v\left(\cdot, t_{n}\right) \circ X^{n, n+1}$, we obtain the semidiscrete Lagrangian scheme

(8)

$$
\left\{\begin{array}{l}
\frac{v\left(x, t_{n+1}\right)-v\left(\cdot, t_{n}\right) \circ X^{n, n+1}}{\Delta t}+\nabla p\left(x, t_{n+1}\right)-\nu \Delta v\left(x, t_{n+1}\right)=f\left(x, t_{n+1}\right), \\
\operatorname{div} v\left(x, t_{n+1}\right)=0, \\
\left.v\left(x, t_{n+1}\right)\right|_{\Gamma}=0 .
\end{array}\right.
$$

The semidiscrete Lagrangian scheme ((8)), which was proposed by Pironneau [23] in combination with finite elements, yields an approximation to the weak solution $(v, p)$ of order $O(\Delta t)$. 


\section{LG methods for Navier-Stokes Equations}

Applying the trapezoidal rule to integrate (7) yields the second order semidiscrete scheme

$$
\left\{\begin{array}{l}
\frac{v\left(x, t_{n+1}\right)-v\left(\cdot, t_{n}\right) \circ X^{n, n+1}}{\Delta t}+\frac{1}{2}\left(p\left(x, t_{n+1}\right)+\mathbf{F}^{-T} \nabla_{X^{n, n+1}} p\left(\cdot, t_{n}\right) \circ X^{n, n+1}\right) \\
=\frac{1}{2} \nu \Delta v\left(x, t_{n+1}\right)+\frac{\nu}{2 J} \operatorname{div}\left(J \mathbf{F}^{-1} \mathbf{I F}^{-T} \nabla_{X^{n, n+1}} v\left(\cdot, t_{n}\right) \circ X^{n, n+1}(x)\right) \\
+\frac{1}{2}\left(f\left(x, t_{n+1}\right)+f\left(\cdot, t_{n}\right) \circ X^{n, n+1}\right), \\
\operatorname{div} v\left(x, t_{n+1}\right)=0, \\
\left.v\left(x, t_{n+1}\right)\right|_{\partial D}=0,
\end{array}\right.
$$

here, $J:=J\left(x, t_{n+1} ; t_{n}\right)$ and $\mathbf{F}^{-T}=\mathbf{F}^{-T}\left(x, t_{n+1} ; t_{n}\right)$. A variant of this scheme combined with finite elements has been studied in [21]. We can also derive a second order scheme by applying the Backward Differentiation Formula of order 2 (BDF2) for the time discretization of (7); thus, setting

$$
d_{t} g\left(y, t_{n+1}\right):=\frac{3 g\left(y, t_{n+1}\right)-4 g\left(y, t_{n}\right)+g\left(y, t_{n-1}\right)}{2 \Delta t},
$$

it follows the second order semidiscrete Lagrangian scheme:

$$
\left\{\begin{array}{l}
d_{t} \bar{v}\left(y, t_{n+1}\right)=\bar{G}\left(y, t_{n+1}\right), \\
\frac{1}{J} \operatorname{div}\left(J \mathbf{F}^{-1} \bar{v}\left(y, t_{n+1}\right)\right)=0, \\
\left.\bar{v}\left(y, t_{n+1}\right)\right|_{\partial D}=0
\end{array}\right.
$$

or equivalently

$$
\left\{\begin{array}{l}
\mathcal{D} v\left(x, t_{n+1}\right)+\nabla p\left(x, t_{n+1}\right)=\nu \Delta v\left(x, t_{n+1}\right)+f\left(x, t_{n+1}\right), \\
\operatorname{div} v\left(x, t_{n+1}\right)=0 \\
\left.v\left(x, t_{n+1}\right)\right|_{\partial D}=0
\end{array}\right.
$$

where

$$
\mathcal{D} v\left(x, t_{n+1}\right):=\frac{3 v\left(x, t_{n+1}\right)-4 v\left(\cdot, t_{n}\right) \circ X^{n, n+1}+v\left(\cdot, t_{n-1}\right) \circ X^{n-1, n+1}}{2 \Delta t} .
$$

This scheme has been used in $[7,9,19]$, and recently in [4,5] and [11]. 


\section{R. Bermejo, L. Saavedra}

\section{First and second order in time LG methods}

Hereafter, we shall focus on the finite element formulation of the semidiscrete schemes $((8))$ and $((9))$, they will be dented by LG-BDF1 and LG-BDF2 methods, respectively. Let $\bar{\Omega}_{h}=\bigcup_{j=1}^{N E} T_{j}$ be a quasi-uniform triangulation of the region $\bar{\Omega}$, here $T_{j}$ denotes a simplex of dimension $d$ the diameter of which is $h_{j}, N E \in \mathbb{N}(N E>1)$ is the number of elements in $\bar{\Omega}_{h}$, and $h=\max _{j} h_{j}$. In relation with $\bar{\Omega}_{h}$, we consider the reference element $\widehat{T}:=\left\{\widehat{x} \in \mathbb{R}^{d}: 0 \leq x_{i} \leq 1,1-\sum_{i=1}^{d} x_{i} \geq 0\right\}$, such that for each $T_{j}$ there exists an invertible mapping $F_{j}: \widehat{T} \rightarrow T_{j}$ of class $C^{1}$, and the $H^{1}$-conforming finite element spaces $\mathbf{V}_{h} \subset H^{1}(\Omega), \mathbf{X}_{h}=\mathbf{V}_{h} \cap \mathbf{H}_{0}^{1}(\Omega)$ and $M_{h} \subset L_{0}^{2}(\Omega)$; we assume that the pair of finite element spaces $\left(\mathbf{X}_{h}, M_{h}\right)$ is inf-sup stable and the following approximation properties hold: for $v \in$ $\mathbf{H}^{s+1}(\Omega) \cap \mathbf{H}_{0}^{1}(\Omega), p \in H^{s_{1}+1}(\Omega), 0 \leq s_{1} \leq m_{1}$ and $0 \leq s \leq m$,

$$
\inf _{v_{h} \in \mathbf{X}_{h}}\left(\left\|v-v_{h}\right\|_{\mathbf{L}^{2}(\Omega)}+h\left\|v-v_{h}\right\|_{\mathbf{H}^{1}(\Omega)}\right) \leq C h^{s+1}\|v\|_{\mathbf{H}^{s+1}(\Omega)}
$$

and

$$
\inf _{q_{h} \in M_{h}}\left(\left\|p-q_{h}\right\|_{L^{2}(\Omega)}+h\left\|p-q_{h}\right\|_{H^{1}(\Omega)}\right) \leq C h^{s_{1}+1}\|p\|_{H^{s_{1}+1}(\Omega)},
$$

where $m$ and $m_{1}$ denote the degree of the polynomials of $\mathbf{X}_{h}$ and $M_{h}$ respectively. We also assume that the following inverse properties hold in $\mathbf{X}_{h}$ : for $0 \leq k \leq m \leq 1$ and $1 \leq p \leq q \leq \infty$,

$$
\left\|v_{h}\right\|_{\mathbf{W}^{m, q}(\Omega)} \leq C h^{d / q-d / p+k-m}\left\|v_{h}\right\|_{\mathbf{W}^{k, p}(\Omega)}
$$

and

$(10 \mathrm{~d})$

$$
\left\|v_{h}\right\|_{\mathbf{L}^{\infty}(\Omega)} \leq D(h)\left\|v_{h}\right\|_{\mathbf{H}^{1}(\Omega)} ; \quad D(h):=\left\{\begin{array}{l}
C\left(1+|\log h|^{1 / 2}\right) \text { if } d=2, \\
C h^{-1 / 2} \text { if } d=3 .
\end{array}\right.
$$

The formulation of LG-BDF1 and LG-BDF2 methods is as follows.

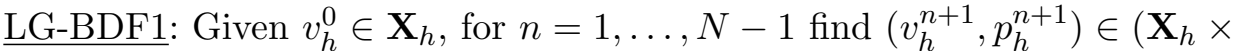
$M_{h}$ ), such that for any $u_{h} \in \mathbf{X}_{h}$ and $q_{h} \in M_{h}$ :

$$
\left\{\begin{array}{l}
\left(v_{h}^{n+1}, u_{h}\right)+\Delta t \nu\left(\nabla v_{h}^{n+1}, \nabla u_{h}\right)-\Delta t\left(p_{h}^{n+1}, \operatorname{div} u_{h}\right) \\
=\left(v_{h}^{n} \circ X_{h}^{n, n+1}, u_{h}\right)+\Delta t\left(f^{n+1}, u_{h}\right), \\
\left(\operatorname{div} v_{h}^{n+1}, q_{h}\right)=0 .
\end{array}\right.
$$




\section{LG methods for Navier-Stokes Equations}

LG-BDF2: Given $v_{h}^{0}, v_{h}^{1} \in \mathbf{X}_{h}$, for $n=1, \ldots, N-1$ find $\left(v_{h}^{n+1}, p_{h}^{n+1}\right) \in$ $\left(\mathbf{X}_{h} \times M_{h}\right)$, such that for any $u_{h} \in \mathbf{X}_{h}$ and $q_{h} \in M_{h}$ :

$$
\left\{\begin{array}{l}
\left(\frac{3 v_{h}^{n+1}}{2}, u_{h}\right)+\Delta t \nu\left(\nabla v_{h}^{n+1}, \nabla u_{h}\right)-\Delta t\left(p_{h}^{n+1}, \operatorname{div} u_{h}\right) \\
=\left(2 v_{h}^{n} \circ X_{h}^{n, n+1}, u_{h}\right)-\left(\frac{1}{2} v_{h}^{n-1} \circ X_{h}^{n-1, n+1}, u_{h}\right)+\Delta t\left(f^{n+1}, u_{h}\right), \\
\left(\operatorname{div} v_{h}^{n+1}, q_{h}\right)=0 .
\end{array}\right.
$$

Since

$$
\left(v_{h}^{n-l} \circ X_{h}^{n-l, n+1}, u_{h}\right)=\sum_{j} \int_{T_{j}} v_{h}^{n-l}\left(X_{h}^{n-l, n+1}(x)\right) \cdot u_{h}(x) d x, l=0,1,
$$

then the calculation of $\int_{T_{j}} v_{h}^{n-l}\left(X_{h}^{n-l, n+1}(x)\right) \cdot u_{h}(x) d x$ and $X_{h}^{n-l, n+1}(x)$ are key issues of LG methods. On the other hand, we notice that $v_{h}^{0}$ in (11), and $v_{h}^{0}$ and $v_{h}^{1}$ in (12) are needed to perform the methods. To this respect, $v_{h}^{0}$ is calculated as the elliptic projection of $v^{0}$ onto $\mathbf{X}_{h}$, that is,

$$
\left(\nabla v_{h}^{0}, \nabla u_{h}\right)=\left(\nabla v^{0}, \nabla u_{h}\right) \text { for all } u_{h} \in \mathbf{X}_{h}
$$

As for the calculation of $\left(v_{h}^{1}, p_{h}^{1}\right)$, we define in $[0, \Delta t]$ the uniform partition $0=t_{0}^{*}<t_{1}^{*}<\ldots<t_{m_{0}}^{*}=\Delta t$ of step $\Delta t^{*}=\frac{\Delta t}{m_{0}}$, such that $\frac{\Delta t^{2}}{m_{0}}=C \Delta t^{3}$, where $C$ is a constant of moderate size; then we calculate the sequence $\left\{\bar{v}_{h}^{k}, \bar{p}_{h}^{k}\right\}_{k=1}^{m_{0}}$ by (11) and set $p_{h}^{1}=\bar{p}_{h}^{m_{0}}, v_{h}^{1}=\bar{v}_{h}^{m_{0}}$. If $v^{0}=0$ in $\Omega$, then we calculate $\left(v_{h}^{1}, p_{h}^{1}\right)$ by solving time dependent Stokes problems with BDF1 as time marching scheme.

For any $x \in \Omega$, the points $X_{h}^{n-l, n+1}(x)(l=0,1)$ are numerical solutions at time instants $t_{n-l}$ of the initial value problem

$$
\left\{\begin{array}{l}
\frac{d X_{h}\left(x, t_{n+1} ; t\right)}{d t}=v_{h}\left(X_{h}\left(x, t_{n+1} ; t\right), t\right), \quad t_{n-l} \leq t<t_{n+1} \\
X_{h}\left(x, t_{n+1} ; t_{n+1}\right)=x
\end{array}\right.
$$

where $v_{h}(\cdot, t)$ is usually calculated by some extrapolation/interpolation formula of the values $v_{h}^{n}$ and $v_{h}^{n-1}$. Noting that for all $n, v_{h}^{n}$ and $v_{h}^{n-1}$ are in $\mathbf{W}^{1, \infty}(D)$, then there is a unique solution $X_{h}\left(x, t_{n+1} ; t\right)$ to $(14)$. 
3.1. Calculation of $\int_{T_{j}} v_{h}^{n-l}\left(X_{h}^{n-l, n+1}(x)\right) \cdot u_{h}(x) d x, l=0,1$

The evaluation of the element integrals is usually done numerically by applying a quadrature rule of high order so as to maintain both the stability and the accuracy that the method would possess if the integrals were calculated exactly. Thus, noting that for $x \in T_{j}, X_{h}^{n-l, n+1}(x)$ is in some $T_{i}$ of the mesh, and letting $u_{h}(x)$ be the $p$ th basis function of $T_{j}$, i.e., $\varphi_{p}^{(j)}$, and ne be the number of velocity nodes per element, we can set

$$
\int_{T_{j}} v_{h}^{n-l}\left(X_{h}^{n-l, n+1}(x)\right) \cdot u_{h}(x) d x=\sum_{k=1}^{n e} V_{k(i)}^{n-l} \int_{T_{j}} \varphi_{k}^{(i)}\left(X_{h}^{n-l, n+1}(x)\right) \varphi_{p}^{(j)}(x) d x,
$$

where $k(i)$ denotes the global number of the node of the mesh $\bar{\Omega}_{h}$ that is the $k$ th node of $T_{i}$, and $\left\{\varphi_{k}^{(i)}\right\}_{k=1}^{n e}$ is the set of local basis functions for the element $T_{i}$. Now, assuming that $X_{h}^{n-l, n+1}(x) \in T_{i}$

$$
\int_{T_{j}} \varphi_{k}^{(i)}\left(X_{h}^{n-l, n+1}(x)\right) \varphi_{p}^{(j)}(x) d x=\int_{\widehat{T}} \widehat{\varphi}_{k}(\widehat{z}) \widehat{\varphi}_{p}(\widehat{x})\left|\frac{\partial F_{j}}{\partial \widehat{x}}\right| d \widehat{x},
$$

where $\widehat{z}:=F_{i}^{-1} \circ X_{h}^{n-l, n+1}(x)$ and $\left\{\widehat{\varphi}_{i}\right\}_{i=1}^{n e}$ is the set of basis functions for the reference element $\widehat{T}$. Finally, we approximate the integrals over $\widehat{T}$ by high order quadrature rules as

$$
\int_{\widehat{T}} \widehat{\varphi}_{k}(\widehat{z}) \widehat{\varphi}_{p}(\widehat{x})\left|\frac{\partial F_{j}}{\partial \widehat{x}}\right| d \widehat{x} \simeq \operatorname{meas}\left(T_{j}\right) \sum_{g=1}^{n q p} \varpi_{g} \widehat{\varphi}_{k}\left(\widehat{z}_{g}\right) \widehat{\varphi}_{p}\left(\widehat{x}_{g}\right)\left|\frac{\partial F_{j}\left(\widehat{x}_{g}\right)}{\partial \widehat{x}}\right|,
$$

where $n q p$ denotes the number of weights, $\varpi_{g}$, and points, $\widehat{x}_{g}$, of the quadrature rule. An algorithmic presentation of the procedure is as follows.

For $j=1,2 \ldots N E\left(N E\right.$ is the number of the elements in $\left.\bar{\Omega}_{h}\right)$

For $l=0,1$

For $k=1,2 \ldots n q p$

(a) Calculate $x_{k}=F_{j}\left(\widehat{x}_{k}\right)$ and then calculate $X_{h}^{n-l, n+1}\left(x_{k}\right)$ by solving (14) with initial condition $x_{k}$.

(b) Find the element $T_{i}$ containing the point $X_{h}^{n-l, n+1}\left(x_{k}\right)$ and calculate $\widehat{z}_{k}=F_{i}^{-1}\left(X_{h}^{n-l, n+1}\left(x_{k}\right)\right)$.

(c) Calculate

$$
v_{h}^{n-l}\left(X_{h}^{n-l, n+1}\left(x_{k}\right)\right)=\sum_{m=1}^{n e} V_{m(i)}^{n-l} \widehat{\varphi}_{m}\left(\widehat{z}_{k}\right)
$$




\section{LG methods for Navier-Stokes Equations}

For $p=1, \ldots n e$

Calculate

$$
\operatorname{meas}\left(T_{j}\right) \sum_{k=1}^{n q p} \varpi_{k} v_{h}^{n-l}\left(X^{n, n+1}\left(x_{k}\right)\right) \widehat{\varphi}_{p}\left(\widehat{x}_{k}\right)\left|\frac{\partial F_{j}\left(\widehat{x}_{k}\right)}{\partial \widehat{x}}\right| .
$$

Assemble these values into a right hand side column vector.

End

3.2. Calculation of the approximate departure points $X_{h}^{n-l, n+1}(x)$

The first thing we should notice is that for all $t_{n}, n>1, v_{h}^{n+1}$ and $X_{h}^{n-l, n+1}$ depend on each other, so a way to proceed avoiding fixed point considerations is to use an explicit procedure to calculate $X_{h}^{n-l, n+1}$. Furthermore, the numerical solution to ((14)) should be calculated by a method of order equal to or larger than the order of the backward scheme employed to discretize the term $\frac{D v}{D t}$. The numerical solution to (14) is frequently calculated by explicit Runge-Kutta schemes of order two or higher, see for instance, [12], [7], [19], [30]. More recently, and based on the good properties of the fixed point implicit multi-step method of order 2 proposed in [29], we have developed and adaptive version of this method that works very well. We first describe a Runge-Kutta scheme of order 2

\section{A Runge-Kutta method of order 2}

For $n=0$, calculate

$$
\left\{\begin{array}{l}
K_{1}=v_{h}^{0}(x) \\
K_{2}=v_{h}^{0}\left(x-\Delta t K_{1}\right) \\
X_{h}^{0,1}(x)=x-\Delta t\left(\frac{K_{1}}{2}+\frac{K_{2}}{2}\right) .
\end{array}\right.
$$

For $n=1,2 \ldots, N-1$, and $l=0$

$$
\left\{\begin{array}{l}
K_{1}=\bar{v}_{h}^{n+1}(x) \\
K_{2}=v_{h}^{n}\left(x-\Delta t K_{1}\right) \\
X_{h}^{n, n+1}(x)=x-\Delta t\left(\frac{K_{1}}{2}+\frac{K_{2}}{2}\right) .
\end{array}\right.
$$

When $l=1$, calculate

$$
\left\{\begin{array}{l}
K_{1}=v_{h}^{n}\left(X_{h}^{n, n+1}(x), t_{n}\right), \\
K_{2}=v_{h}^{n-1}\left(X_{h}^{n, n+1}(x)-\Delta t K_{1}\right), \\
X_{h}^{n-l, n+1}(x)=X_{h}^{n, n+1}(x)-\Delta t\left(\frac{K_{1}}{2}+\frac{K_{2}}{2}\right) .
\end{array}\right.
$$


Here,

$$
\bar{v}_{h}^{n+1}(\cdot)=2 v_{h}^{n}(\cdot)-v_{h}^{n}(\cdot),
$$

is a second order approximation to $v_{h}^{n+1}$. In this algorithm the crucial steps are the calculations of $K_{1}$ and $K_{2}$. Noting that $v_{h}$ is known only at the mesh points $\left\{x_{i}\right\}$ at time steps $t_{n}, t_{n-1} \ldots, t_{0}$, then it follows that, in general, $v_{h}^{n}(y)$ and $v_{h}^{n}\left(y-\Delta t K_{1}\right)$ are unknowns because both $y$ and $y-\Delta t K_{1}$ do not coincide with mesh points, so they are calculated by finite element interpolation on the elements where the points $y$ and $y-\Delta t K_{1}$ are located. A search-locate algorithm to identify such elements and simultaneously perform the finite element interpolations is described in [2]. The algorithm also informs if the points lie outside the domain.

Remark 3.1. For all $x \in \Omega$, the departure points $X_{h}^{n-l, n+1}(x)$ cannot leave the computational domain through the solid boundaries because on such boundaries either $v_{h}=0$ or $v_{h} \cdot \mathbf{n}=0, \mathbf{n}$ being the unit outward normal, so that it can be proved that the trajectories of (14) cannot cross the solid boundaries. However, in many cases, in particular when $\Delta t$ is not small enough and points $y$ are in elements close to the solid boundaries of $\Omega$, the numerical errors may cause some points $y-\Delta t K_{1}$ to be outside the computational domain. One way to alleviate this trouble is presented in the following adaptive algorithm.

\section{An adaptive fixed point implicit multi-step method of order 2}

We present the algorithm for the calculation of the points $X_{h}^{n, n+1}(x)$ because, as we have said above, we calculate $X_{h}^{n-1, n+1}(x)$ in two steps: first, we compute $X_{h}^{n, n+1}(x)$, and then $X_{h}^{n-1, n} \circ X_{h}^{n, n+1}(x)$. For the sake of clarity, we temporally go back to the notation $X_{h}\left(x, t_{n+1} ; t_{n}\right)$ and present the non-adaptive version of the algorithm first. Since the solution of (14)) can be expressed by the formula

$$
X_{h}\left(x, t_{n+1} ; t_{n}\right)=x-\int_{t_{n}}^{t_{n+1}} v_{h}\left(X_{h}\left(x, t_{n+1} ; t\right), t\right) d t,
$$

then, setting

$$
\alpha=\int_{t_{n}}^{t_{n+1}} v_{h}\left(X_{h}\left(x, t_{n+1} ; t\right), t\right) d t=x-X_{h}\left(x, t_{n+1} ; t_{n}\right)
$$

and evaluating the integral by the mid-point rule, we obtain a formula to approximate $\alpha$ up to order $O\left(\Delta t^{3}\right)$ such as

$$
\alpha=\Delta t v_{h}\left(X\left(x, t_{n+1} ; t_{n}+\frac{\Delta t}{2}\right), t_{n}+\frac{\Delta t}{2}\right) .
$$




\section{LG methods for Navier-Stokes Equations}

Moreover, using the second order approximation

$$
X_{h}\left(x, t_{n+1} ; t_{n}+\frac{\Delta t}{2}\right) \simeq \frac{1}{2}\left(x+X_{h}\left(x, t_{n+1} ; t_{n}\right)\right)=x-\frac{\alpha}{2},
$$

the second order extrapolation

$$
v_{h}\left(\cdot, t_{n}+\frac{\Delta t}{2}\right)=\frac{3 v_{h}\left(\cdot, t_{n}\right)}{2}-\frac{v_{h}\left(\cdot, t_{n-1}\right)}{2},
$$

and defining the operator $G: \Omega \rightarrow \Omega$ as

$$
G(\alpha)=\frac{\Delta t}{2}\left(3 v_{h}\left(x-\frac{\alpha}{2}, t_{n}\right)-v_{h}\left(x-\frac{\alpha}{2}, t_{n-1}\right)\right),
$$

we can calculate $\alpha$ as the fixed point of the operator $G$ if

$$
\Delta t \max _{\left(x, t_{n}\right) \in B_{j} \times(0, T)}\left|\nabla v_{h}^{n}(x)\right|<2,
$$

because under this condition $G$ is a contractive operator. $B_{j}$ is a neighborhood of the point $x$ such that $X_{h}^{n, n+1}(x) \in B_{j}$. A fixed point iterative procedure to calculate $\alpha$ at time $t_{n+1}$ is the following.

Given $\epsilon$, a real number such that $0<\epsilon \ll 1, k_{\max } \in \mathbb{N}\left(k_{\max } \geq 1\right)$, $v_{h}^{n}$ and $v_{h}^{n-1}$ :

(1) Set

$$
\alpha^{(0)}=\Delta t\left(\frac{3}{2} v_{h}^{n}(x)-\frac{1}{2} v_{h}^{n-1}(x)\right) .
$$

(2) For $k=0,1, \ldots, k_{\max }$

$$
\alpha^{(k+1)}=\Delta t\left(\frac{3}{2} v_{h}^{n}\left(x-\frac{1}{2} \alpha^{(k)}\right)-\frac{1}{2} v_{h}^{n}\left(x-\frac{1}{2} \alpha^{(k)}\right)\right) .
$$

The iterative procedure stops when $k \leq k \max$ and

$$
\frac{\left|\alpha^{(k+1)}-\alpha^{(k)}\right|}{\left|\alpha^{(k)}\right|} \leq \varepsilon
$$

(3) Set

$$
X_{h}^{n, n+1}(x)=x-\alpha^{(k+1)} .
$$

If $\Delta t$ is so large that either the iterative procedure does not converge or the point $X_{h}^{n, n+1}(x)$ lies outside the domain, then we successively halve 


\section{R. Bermejo, L. Saavedra}

$m$ times $\Delta t$ until convergence or $X_{h}^{n, n+1}(x)$ is inside the domain; thus, this yields and adaptive fixed point iterative procedure. To describe such a procedure, we consider that $\Delta t \rightarrow \frac{\Delta t}{2^{m}}, m \geq 0$, then $X_{h}\left(x, t_{n+1}, t_{n+1}-\frac{\Delta t}{2^{m}}\right)$ is given by

$$
X_{h}\left(x, t_{n+1}, t_{n+1}-\frac{\Delta t}{2^{m}}\right)=x-\int_{t_{n+1}-\frac{\Delta t}{2^{m}}}^{t_{n+1}} v_{h}\left(X_{h}\left(x, t_{n+1}, t\right), t\right) d t \equiv x-\alpha .
$$

Noting that

$$
\begin{aligned}
& \int_{t_{n+1}-\frac{\Delta t}{2^{m}}}^{t_{n+1}} v_{h}\left(X_{h}\left(x, t_{n+1}, t\right), t\right) d t= \\
& =\frac{\Delta t}{2^{m}} v_{h}\left(X_{h}\left(x, t_{n+1}, t_{n+1}-\frac{\Delta t}{2^{m+1}}\right), t_{n+1}-\frac{\Delta t}{2^{m+1}}\right)+O\left(\left(\frac{\Delta t}{2^{m}}\right)^{3}\right),
\end{aligned}
$$

and

$$
X_{h}\left(x, t_{n+1}, t_{n+1}-\frac{\Delta t}{2^{m+1}}\right)=\frac{1}{2}\left(x+X_{h}\left(x, t_{n+1}, t_{n+1}-\frac{\Delta t}{2^{m}}\right)\right)+O\left(\left(\frac{\Delta t}{2^{m}}\right)^{2}\right),
$$

it follows that

$$
\begin{aligned}
& X_{h}\left(x, t_{n+1}, t_{n+1}-\frac{\Delta t}{2^{m}}\right) \\
& =x-\frac{\Delta t}{2^{m}} v_{h}\left(X_{h}\left(x, t_{n+1}, t_{n+1}-\frac{\Delta t}{2^{m+1}}\right), t_{n+1}-\frac{\Delta t}{2^{m+1}}\right)+O\left(\left(\frac{\Delta t}{2^{m}}\right)^{3}\right),
\end{aligned}
$$

and

$$
X_{h}\left(x, t_{n+1}, t_{n+1}-\frac{\Delta t}{2^{m+1}}\right)=x-\frac{\alpha}{2}+O\left(\left(\frac{\Delta t}{2^{m}}\right)^{2}\right) .
$$

Now, making use of the extrapolation formula

$$
v_{h}^{n+1-2^{-m-1}}=\left(2-2^{-m-1}\right) v_{h}^{n}-\left(1-2^{-m-1}\right) v_{h}^{n-1}+O\left(\left(\frac{\Delta t}{2^{m}}\right)^{2}\right)
$$

we can set

$$
\begin{array}{r}
X_{h}\left(x, t_{n+1}, t_{n+1}-\frac{\Delta t}{2^{m}}\right)=x-2^{-m} \Delta t\left(\left(2-2^{-m-1}\right) v_{h}^{n}\right. \\
\left.-\left(1-2^{-m-1}\right) v_{h}^{n-1}\right)\left(x-\frac{\alpha}{2}\right)+O\left(\left(\frac{\Delta t}{2^{m}}\right)^{3}\right)
\end{array}
$$

Thus, neglecting $O\left(\left(\frac{\Delta t}{2 m}\right)^{3}\right)$ terms and defining $G^{m}: \Omega \rightarrow \Omega$ as

$$
G^{m}(\alpha)=2^{-m} \Delta t\left(\left(2-2^{-m-1}\right) v_{h}^{n}-\left(1-2^{-m-1}\right) v_{h}^{n-1}\right)\left(x-\frac{\alpha}{2}\right),
$$




\section{LG methods for Navier-Stokes Equations}

it follows that

$$
\alpha=G^{m}(\alpha) .
$$

Thus, after calculating $\alpha$ as the fixed point of $G^{m}$, we use (18) and obtain $X_{h}\left(x, t_{n+1}, t_{n}\right)$ by performing the following backward procedure: for $\mathrm{i}=\mathrm{m}-1$, $\mathrm{m}-2, \ldots 0$

$$
\begin{gathered}
X_{h}\left(x, t_{n+1}, t_{n+1}-\frac{\Delta t}{2^{i}}\right)=x-2^{-i} \Delta t\left(2-2^{-i-1}\right) v_{h}^{n}\left(X_{h}\left(x, t_{n+1}, t_{n+1}-\frac{\Delta t}{2^{i+1}}\right)\right) \\
-2^{-i} \Delta t\left(1-2^{-i-1}\right) v_{h}^{n-1}\left(\left(X_{h}\left(x, t_{n+1}, t_{n+1}-\frac{\Delta t}{2^{i+1}}\right)\right) .\right.
\end{gathered}
$$

An algorithmic description of the adaptive fixed point iterative procedure is as follows:

$0<m \leq M_{\max } \in \mathbb{N}$

Setting $m=1$, then

(1) Calculate

$$
\alpha^{(0)}=2^{-m+1} \Delta t\left(\frac{3}{2} v_{h}^{n}(x)-\frac{1}{2} v_{h}^{n-1}(x)\right) .
$$

If $x-\frac{1}{2} \alpha^{(0)}$ leaves the computational domain through a solid boundary, set $m=m+1$ and repeat (1).

(2) For $k=0,1, \ldots, k_{\max }$ calculate

$$
\alpha^{(k+1)}=2^{-m+1} \Delta t v_{h}\left(x-\frac{1}{2} \alpha^{(k)}, t_{n+1}-\frac{\Delta t}{2^{m}}\right) .
$$

(a) If $x-\frac{1}{2} \alpha^{(k+1)}$ leaves the computational domain through a solid boundary, set $m=m+1$ and repeat (1).

(b) If $k=k_{\max }$ and

$$
\frac{\left|\alpha^{(k+1)}-\alpha^{(k)}\right|}{\left|\alpha^{(k)}\right|}>\varepsilon,
$$

then set $m=m+1$ and repeat (1).

(c) If

$$
\frac{\left|\alpha^{(k+1)}-\alpha^{(k)}\right|}{\left|\alpha^{(k)}\right|} \leq \varepsilon
$$

stop the iterations.

(3) Set

$$
X_{h}\left(x, t_{n+1} ; t_{n+1}-\frac{\Delta t}{2^{m}}\right)=x-\alpha^{(k+1)}\left(t_{n+1}-\frac{\Delta t}{2^{m}}\right) .
$$




\section{R. Bermejo, L. Saavedra}

(3) For $i=m-1, . ., 0$ calculate

$$
\begin{aligned}
& X_{h}\left(x, t_{n+1} ; t_{n+1}-\frac{\Delta t}{2^{i}}\right) \\
& =x-2^{-i} \Delta t v_{h}\left(X_{h}\left(x, t_{n+1} ; t_{n+1}-\frac{\Delta t}{2^{i+1}}\right), t_{n+1}-\frac{\Delta t}{2^{i+1}}\right) .
\end{aligned}
$$

Note that when $i=0, X_{h}^{n, n+1}(x)=X_{h}\left(x, t_{n+1} ; t_{n}\right)$. In the above formula

$$
v_{h}\left(\cdot, t_{n+1}-\frac{\Delta t}{2^{k}}\right)=\left(2-2^{-k}\right) v_{h}\left(\cdot, t_{n}\right)-\left(1-2^{-k}\right) v_{h}\left(\cdot, t_{n-1}\right), 0 \leq k \leq m .
$$

It may happen that despite the adaptive algorithm there are still some points leaving the domain, we artificially move these points to the barycenter of the elements through which they are leaving the domain. It can be shown that the adaptive iterative procedure converges if

$$
\Delta t \max _{\left(x, t_{n}\right) \in B_{j} \times(0, T)}\left|\nabla v_{h}^{n}(x)\right|<2^{m} .
$$

\section{Substepping}

Another second order scheme, termed substepping, was proposed by Buscaglia and Dari (1992) with the idea of not leaving points outside the domain. Such an algorithm is implemented in two steps as follows:

1) For $t \in\left[t_{n-l}, t_{n+1}\right)$ set

$$
\frac{d X_{h}\left(x, t_{n+1} ; t\right)}{d t}=\widetilde{v}_{h}\left(X_{h}\left(x, t_{n+1} ; t\right), t\right),
$$

where $\widetilde{v}_{h}\left(X_{h}\left(x, t_{n+1} ; t\right), t\right)$ is an approximation to $v_{h}\left(X_{h}\left(x, t_{n+1}, t\right)\right)$ by the second order extrapolation formula

$$
\widetilde{v}_{h}(\cdot, t)=v_{h}\left(\cdot, t_{n-1}\right)+\frac{t-t_{n-1}}{\Delta t}\left(v_{h}\left(\cdot, t_{n}\right)-v_{h}\left(\cdot, t_{n-1}\right)\right) .
$$

2) Set $N P=1$ and solve $((20))$ by a second order predictor-corrector scheme with step $\Delta t^{*}=\Delta t / N P$; if $X_{h}\left(x, t_{n+1} ; t_{n-l}\right)$ is outside $\Omega$, set $N P=$ $N P+1$ and repeat the procedure until $X_{h}\left(x, t_{n+1} ; t_{n-l}\right) \in \Omega$.

\section{Error bounds for LG-BDF1 and LG-BDF2 methods}

Süli [27] develops a methodology based on mathematical induction on the index $n$ to calculate the error estimates for LG-BDF1 methods, such an approach can be extended to the calculation of the error estimates for LGBDF2 methods as well, see [5]. Recalling that $m$ and $m_{1}$ are the degree of 


\section{LG methods for Navier-Stokes Equations}

the polynomials of $\mathbf{X}_{h}$ and $M_{h}$ respectively, one proves the error estimates by making the following assumptions:

(A1) $v^{0} \in \mathbf{H}^{m+1}(\Omega) \cap \mathbf{V}$,

(A2) $v \in L^{\infty}\left(\mathbf{V} \cap \mathbf{H}^{m+1}(\Omega)\right) \cap C\left(\mathbf{C}^{0,1}(\bar{\Omega})\right)$,

(A3) $v_{t} \in L^{2}\left(\mathbf{V} \cap \mathbf{H}^{m+1}(\Omega)\right), D_{t}^{2} v$ and $D_{t}^{3} v \in L^{2}\left(\mathbf{L}^{2}(\Omega)\right)$,

(A4) $p \in L^{\infty}\left(H^{m_{1}+1}(\Omega) \cap L_{0}^{2}(\Omega) \cap L^{\infty}(\Omega)\right)$ and $p_{t} \in L^{2}\left(H^{m_{1}+1}(\Omega)\right)$;

(A5) the mesh restriction to get optimal error estimates $\Delta t=o\left(h^{d / 4}\right)$;

(A6) for $l=0,1$ there exists a constant $\bar{c}$ indepent of $h$ and $\Delta t$, such that

$$
\left\|v^{l}-v_{h}^{l}\right\|_{\mathbf{L}^{2}(\Omega)}+h\left[\left\|v^{l}-v_{h}^{l}\right\|_{\mathbf{H}^{1}(\Omega)}+\left\|p^{l}-p_{h}^{l}\right\|_{L^{2}(\Omega)}\right]=\bar{c}\left(h^{m+1}+l \Delta t^{3}\right) ;
$$

(A7) (Induction hypothesis) for all $n$, such that $0 \leq n<N$, there exist constants $h_{s}<1$, and $C>0$ independent of $\Delta t, h$ and $n$ such that for $h \in\left(0, h_{s}\right)$

$$
\begin{gathered}
\left\|v-v_{h}\right\|_{l^{\infty}\left(0, t_{n} ; \mathbf{L}^{2}(\Omega)\right)} \leq C\left(h^{m+1}+\Delta t^{\omega}\right), \\
\left\|v-v_{h}\right\|_{l^{\infty}\left(0, t_{n} ; \mathbf{H}^{1}(D)\right)} \leq C\left(h^{m}+\Delta t^{\omega}\right),
\end{gathered}
$$

where $\omega=1$ for $L G$-BF1 and $\omega=2$ for $L G$-BDF2.

Notice that for Taylor-Hood element $\mathbb{P}_{2} / \mathbb{P}_{1}, m=2$ and $m_{1}=1$, and for the mini-element, i.e., the element $\mathbb{P}_{1}$-bubble $/ \mathbb{P}_{1}, m=1$ and $m_{1}=1$. A consequence of the induction hypothesis is that, see [27] and [1], there is a constant $h_{1} \in\left(0, h_{s}\right)$ independent of $\Delta t$ and $n$ such that

$$
\Delta t\left|v_{h}^{n}\right|_{\mathbf{W}^{1, \infty}(D)} \leq \varepsilon_{d}(h)<1 \quad \forall h \in\left(0, h_{1}\right]
$$

Condition (21) allows us to show that the mapping $x \rightarrow X_{h}^{n-l, n+1}$ is, for all $n$, a homeomorphism from $\Omega$ onto $\Omega$.

With these assumptions we have the following result.

Theorem 4.1. Assume that the trajectories are calculated by a numerical method of order $r \geq 2$ and (A1)-(A7), ((10a))-((10d)), and $\Delta t=O\left(h^{\sigma}\right)$ as $h \rightarrow 0$, with $\sigma>\frac{d-1}{2}$, hold. Then there are constants $C$ and $K$ of the form $C=\max \left(\bar{c}, K\left(\nu^{-1}, v, p, T\right) \exp (\kappa T)\right)$, where the constant $\kappa$ depends on $\|\nabla v\|_{L^{\infty}\left(\mathbf{L}^{\infty}\right)}$, such that

$$
\left\{\begin{array}{c}
\left\|v-v_{h}\right\|_{l^{\infty}\left(0, t_{n} ; \mathbf{L}^{2}(D)\right)} \leq C\left(h^{m+1}+\Delta t^{\omega}\right), \\
\left\|v-v_{h}\right\|_{l^{\infty}\left(0, t_{n} ; \mathbf{H}^{1}(D)\right)} \leq C\left(h^{m}+\Delta t^{\omega}\right), \\
\left\|p-p_{h}\right\|_{l^{2}\left(0, t_{n} ; L^{2}(D)\right)} \leq C\left(h^{m_{1}+1}+\Delta t^{\omega}\right)
\end{array}\right.
$$




\section{R. Bermejo, L. Saavedra}

\section{Numerical results}

We illustrate the performance of LG-BDF methods running some benchmark tests of three dimensional flows.

\subsection{Lid-driven cavity flow}

This is a widely used benchmark test to validate numerical schemes. The first accurate solutions for the three dimensional cavity problem were reported by Ku et al.(1987) and Tang et al. (1995) among others; more recent results employing stabilized finite elements for high Reynolds numbers are presented in Hachen et al. (2010). Complex phenomena appear in the cubic cavity and Taylor-Görter like vortices are formed at relatively low Reynolds numbers. We simulate the flow in the cavity, which is plotted in Figure 1, using the time marching LG-BDF2 method with the $\mathbb{P}_{1}-$ bubble $/ \mathbb{P}_{1}$ element and a time step $\Delta t=0.05$, until the flow reaches the steady state; we consider that the steady state is reached when

$$
\frac{\left\|v_{h}^{n+1}-v_{h}^{n}\right\|_{\mathbf{L}^{2}(\Omega)}}{\Delta t} \leq 10^{-6}, \frac{\left\|p_{h}^{n+1}-p_{h}^{n}\right\|_{L^{2}(\Omega)}}{\Delta t} \leq 10^{-6} .
$$

The calculations are carried out with $\mathrm{Re}=1000$, at this value of Re steady vortices are expected. The numerical simulations are performed on a nonuniform mesh of 350085 elements and 64396 vertices, with the particularity that the mesh parameter $h$ takes the value $h=0.01$ near the wall, and then increases as we move towards the center of the cavity. A section of the three-dimensional mesh is displayed in Figure 2.

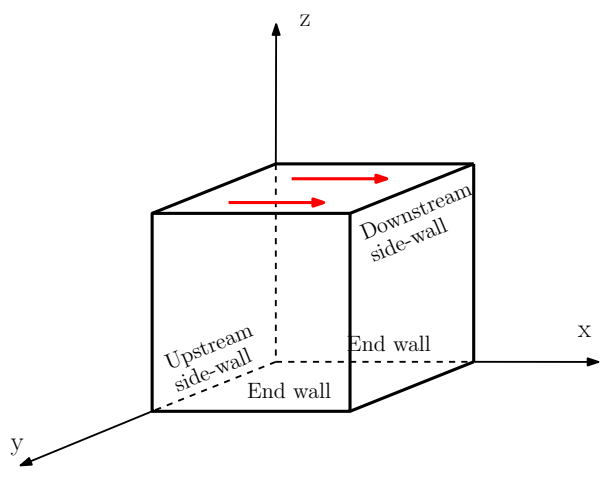

Figure 1. Flow configuration of the $3 \mathrm{D}$ cavity problem

To avoid discontinuities, we follow to Hachem et al. [16] and define the velocity of the moving boundary by the expression

$$
v_{1}=\left(1-x^{18}\right)^{2}\left(1-y^{18}\right)^{2}, v_{2}=0, v_{3}=0 .
$$




\section{LG methods for Navier-Stokes Equations}

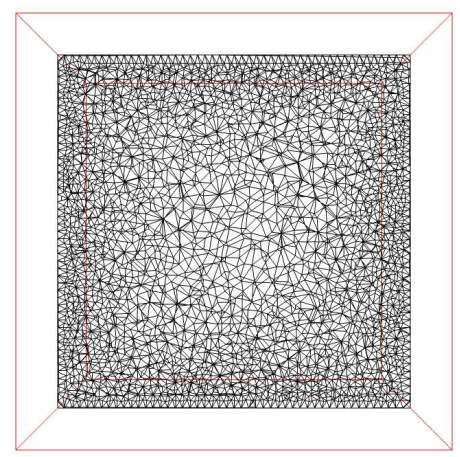

Figure 2. A cross-section of the mesh for the lid-driven cavity at $y=0.5$

The velocity is assumed to be zero on the rest of the walls. The initial condition is $v=0$ in the whole domain. The departure points are calculated by the adaptive fixed point implicit multi-step method of order 2 . The integrals (15) are approximated with a quadrature rule of order 6 (24 points). The steady state is reached after 1354 time steps. Figure 3 shows the projections of velocity vectors on the planes $x=0.5, y=0.5$ and $z=0.5$. In the graphics corresponding to the plane $x=0.5$, we can see the corner eddies caused by the presence of the walls in the planes $y=0$ and $y=1$; moreover, we observe, in the plane $y=0.5$, the existence of the main circulation cell and the downstream secondary eddy formed in the bottom left corner, whereas we can see the Taylor-Göter-like vortices in the plane $z=0.5$. These results are in perfect agreement with those reported in Hachem et al [16] and Tang et al [28].

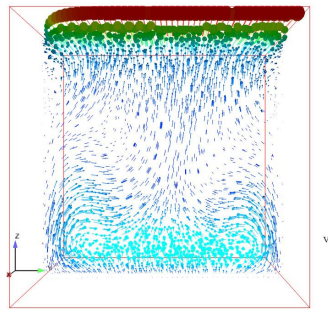

Plane $x=0.5$

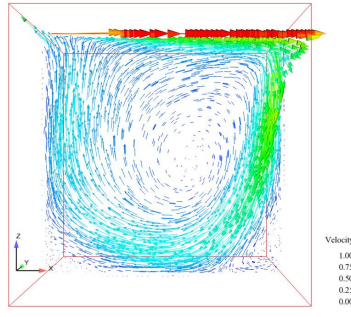

Plane $y=0.5$

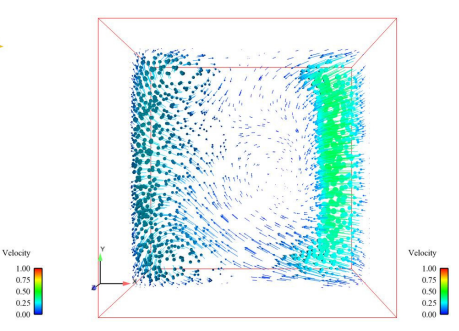

Plane $z=0.5$

Figure 3. Velocity vectors on the three middle planes

The pressure contours on the three middle planes are displayed in Figure 4, they are similar to those obtained by Tang et al [28].

In Figure 5 we represent the profiles of the first component of the velocity at the vertical centerline and the third component of the velocity at 


\section{R. Bermejo, L. Saavedra}

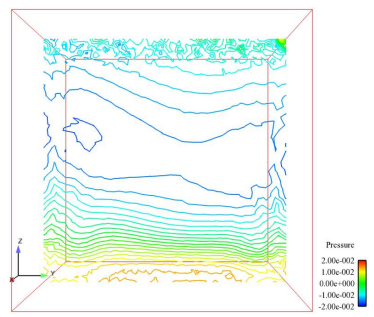

Plane $x=0.5$

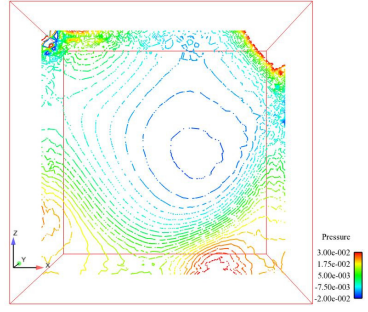

Plane $y=0.5$

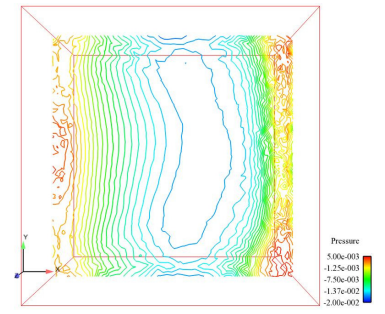

Plane $z=0.5$

Figure 4. Pressure contours on the three middle planes

the horizontal centerline obtained by different methods. We see that our results compare very well with the results of Tang et al [28], Shu et al [26] and Hachem et al [16], although ours have been calculated on a mesh that is coarser than the meshes used in those papers. Also, to add a piece of further information, we include the profiles obtained with the commercial code FLUENT using a second order finite volume method and the same mesh.
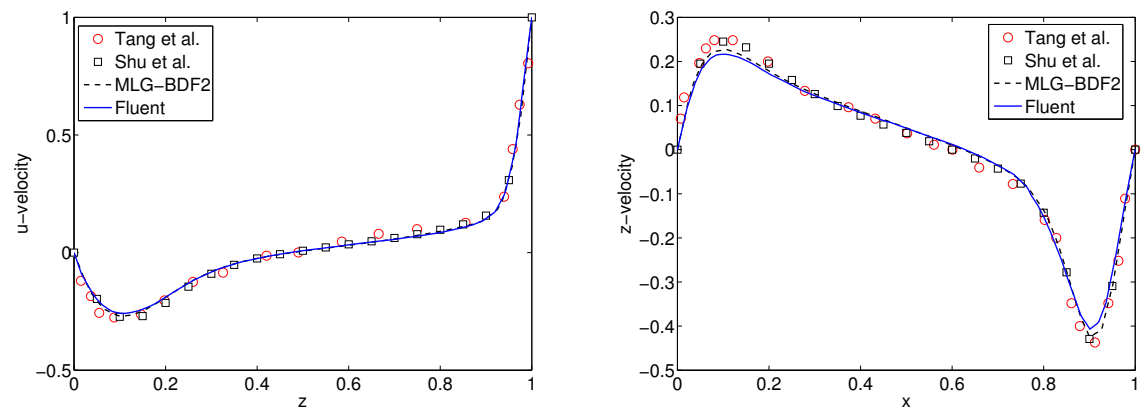

Figure 5. Comparison of velocity profiles $v_{x}$ and $v_{z}$ along central axes on plane $y=0.5$.

\subsection{Three dimensional flow around a cylinder}

We present the results obtained with the LG-BDF2 method using the $\mathbb{P}_{1}$ - bubble $/ \mathbb{P}_{1}$ element for the $3 \mathrm{D}$ stationary incompressible flow around a cylinder, see John [17] for the definition of this example. The geometry of the test is shown in Figure 6. We employ a mesh composed of 476560 elements and 87631 vertices, with the mesh parameter $h$ being equal to 0.005 near the cylinder. A section of the mesh is plotted in Figure 7.

The boundary conditions are zero velocity on the solid boundaries, the inflow velocity is given by

$$
v_{\text {in }}=\left(7.2 x_{1} x_{3}\left(H-x_{2}\right)\left(H-x_{3}\right) / H^{4}, 0,0\right),
$$




\section{LG methods for Navier-Stokes Equations}

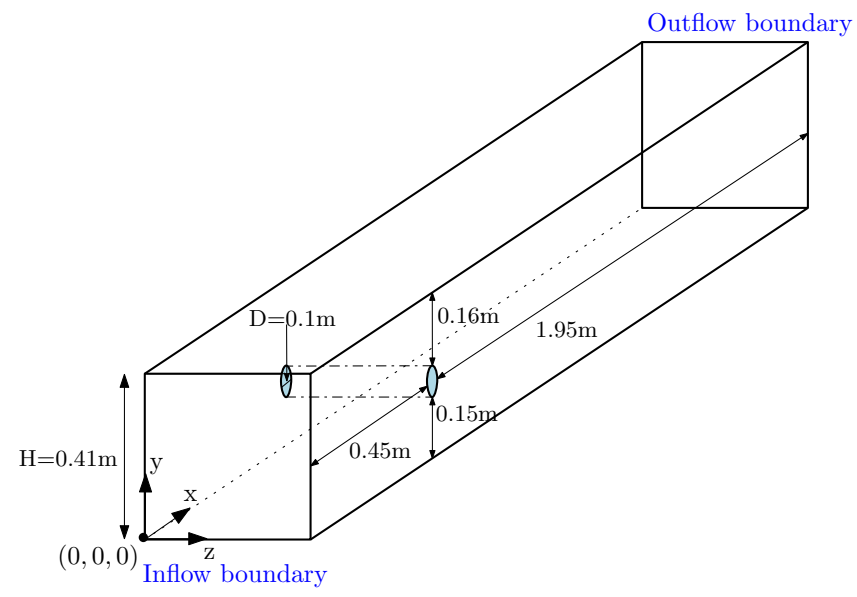

Figure 6. Computational domain

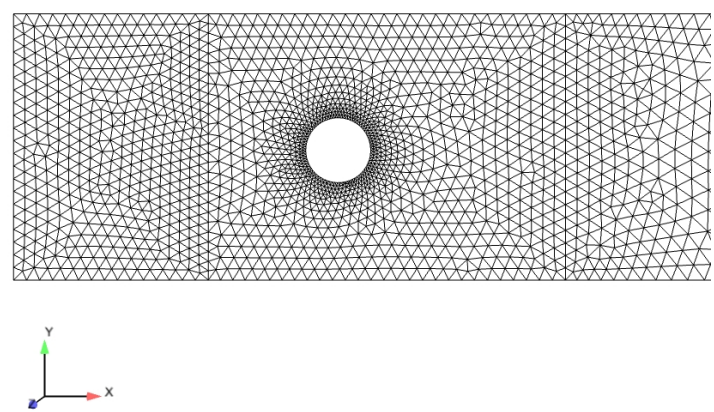

Figure 7. Mesh around the cylinder

where $H=0.41 \mathrm{~m}$ is the height of the channel, and the conventional donothing condition $\left(-\nu \frac{\partial v}{\partial \mathbf{n}}+p \mathbf{n}=0\right)$ on the outflow boundary. The kinematic viscosity of the fluid is $\nu=10^{-3} \mathrm{~m}^{2} / \mathrm{s}$, and $\operatorname{Re}\left(=\frac{v_{i n} D}{\nu}\right)$ is 20 .

The quantities chosen to represent the numerical solution are the pressure difference $\Delta p$ between the points $(0.45,0.2,0.205)$ and $(0.55,0.2,0.205)$, and the drag $\left(c_{d}\right)$ and lift $\left(c_{l}\right)$ coefficients. These coefficients are given by the formulas:

$$
c_{d}=\frac{2}{\rho v_{0}^{2} D H} F_{d}=\frac{500}{0.41} F_{d}, \quad c_{l}=\frac{2}{\rho v_{0}^{2} D H} F_{l}=\frac{500}{0.41} F_{l},
$$

where $F_{d}$ and $F_{l}$ denote the drag and lift forces respectively, which are 


\section{R. Bermejo, L. Saavedra}

expressed as

$$
F_{d}=\int_{C}\left(\nu \frac{\partial\left(v \cdot \tau_{1}\right)}{\partial \mathbf{n}} n_{y}-p n_{x}\right), F_{l}=-\int_{C}\left(\nu \frac{\partial\left(v \cdot \tau_{1}\right)}{\partial \mathbf{n}} n_{x}+p n_{y}\right) .
$$

Here, $C$ denotes the lateral surface of the cylinder, $\mathbf{n}=\left(n_{x}, n_{y}, 0\right)^{T}$ is the inward pointing unit normal vector with respect to $\Omega$, and $\tau_{1}=\left(n_{y},-n_{x}, 0\right)^{T}$ and $\tau_{2}=(0,0,1)^{T}$ are the tangent vectors. We compute the integrals $((26))$ following the method presented in [17], which evaluates the surface integrals as volume integrals over the whole domain. This way of evaluating the integrals is easy to implement and gives more accurate results than the direct numerical calculation of the surface integrals. The results of Table 1 have been calculated with a time step $\Delta t=0.005$, employing the adaptive fixed point implicit multi-step algorithm for the calculation of the departure points. We consider that the steady state is reached when conditions $((23))$ are fulfilled. The Min and Max values in Table 1 define the interval of values for $c_{l}$ and $c_{d}$ according to [25], however, the most accurate values for $c_{l}, c_{d}$ and $\Delta p$ are $c_{d}=6.1853, c_{l}=0.0094$, and $\Delta p=0.1713$, and they are calculated in [8] with a DWR adaptive finite element method using $\mathbb{Q}_{2} / \mathbb{Q}_{2}$ elements and local projection stabilization. We also show in this table the results obtained (with the same mesh as the one used in the LG method) by the commercial software FLUENT using a second order finite volume scheme.

\begin{tabular}{|c||c|c|c|c|}
\hline Coefficients & Min. & Max. & LG & Fluent \\
\hline \hline$c_{d}$ & 6.05 & 6.25 & 6.2172 & 6.1956 \\
\hline$c_{l}$ & 0.008 & 0.01 & 0.0096 & 0.0104 \\
\hline$\Delta p$ & 0.165 & 0.175 & 0.1753 & 0.1637 \\
\hline
\end{tabular}

Finally, we represent in Figure 8 the streamlines on section $y=0.2$; it is worth noting that a vortex is generated behind the cylinder, revealing thus the three-dimensionality of this flow.

All these calculations were carried out on a node with an Intel Xeon processor X5570.

\section{Projection/LG methods}

As we mention in the introductory section, LG methods yield every time step a linear Stokes problem, in the previous numerical tests we solve such Stokes problems by a direct method; however, Achdou and Guermond (2000) and Guermond and Minev (2003) apply LG methods in combination with fractional steps schemes to decouple velocity and pressure in a 


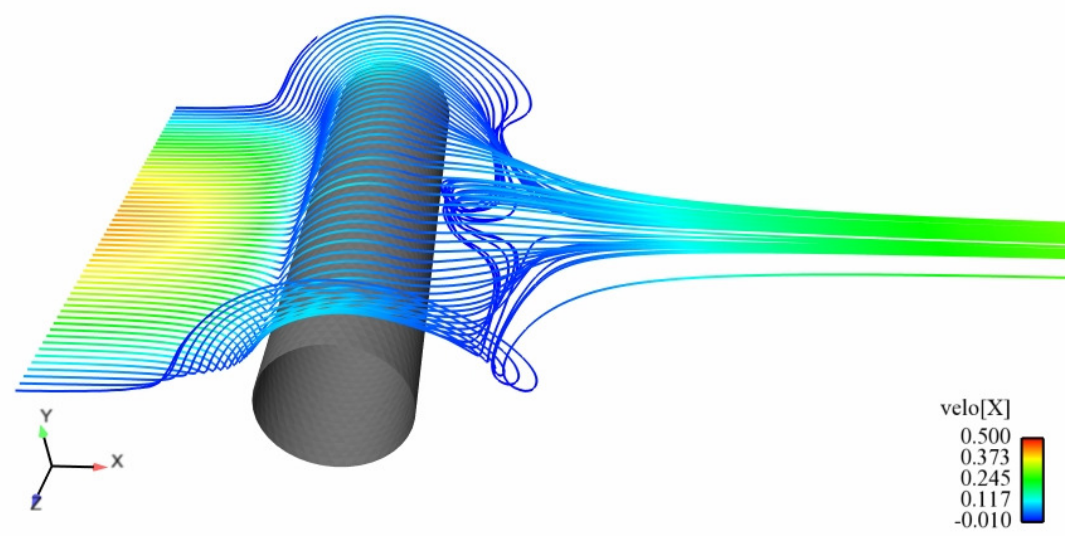

Figure 8. Stream lines at $y=0.2$ colored by $v_{x}$

way that in the first step a velocity is calculated by solving a viscous equation satisfying the boundary conditions, then the pressure is obtained by solving a Poisson equation with homogeneous Neumann boundary conditions if the boundary conditions for the velocity are of Dirichlet type on $\Gamma$ (see [14] for the case in which the velocity is also subject to open boundary conditions), and finally the divergence free velocity is calculated by a projection of the viscous velocity onto a divergence free subspace. Specifically, the projection/LG method of Guermond, Achdou and Minev to calculate a numerical solution to $((1))-((3))$ is the following.

Given $v_{h}^{0} \in \mathbf{X}_{h}$, for $n=0,1 \ldots N-1$ find $\left(\bar{v}_{h}^{n+1}, v_{h}^{n+1}, p_{h}^{n+1}\right)$ such that

$$
\begin{array}{r}
\left(\bar{v}_{h}^{n+1}, u_{h}\right)+\Delta t \nu\left(\nabla \bar{v}_{h}^{n+1}, \nabla u_{h}\right)=\left(v_{h}^{n} \circ X_{h}^{n, n+1}, u_{h}\right)+\Delta t\left(p_{h}^{n}, \operatorname{div} u_{h}\right) \\
+\Delta t\left(f^{n+1}, u_{h}\right), \quad \forall u_{h} \in \mathbf{X}_{h},
\end{array}
$$$$
\left(\nabla\left(p_{h}^{n+1}-p_{h}^{n}\right), \nabla q_{h}\right)=\frac{-1}{\Delta t}\left(\operatorname{div} \bar{v}_{h}^{n+1}, q_{h}\right), \quad \forall q_{h} \in M_{h},
$$$$
v_{h}^{n+1}=\bar{v}_{h}^{n+1}-\Delta t \nabla\left(p_{h}^{n+1}-p_{h}^{n}\right) .
$$

Notice that $\operatorname{div} v_{h}^{n+1}=0$, and the $L^{2}$ projection of this velocity onto $\mathbf{X}_{h}$ is the velocity employed to calculate $X_{h}^{n, n+1}$. Guermond and Minev proved the following result

Theorem 6.1. Under the hypotheses of Theorem 1 there are constants, $c_{e}$, $c_{s}$, independent of $\Delta t$ and $h$, and $h_{s}$ such that for $h \in\left(0, h_{s}\right]$ and $\Delta t=$ 


\section{R. Bermejo, L. Saavedra}

$c_{s} h^{d / 3}$

$$
\begin{gathered}
\left\|v-v_{h}\right\|_{l^{\infty}\left(0, t_{n} ; \mathbf{L}^{2}(D)\right)}+\left\|v-\bar{v}_{h}\right\|_{l^{\infty}\left(0, t_{n} ; \mathbf{L}^{2}(D)\right)} \leq c_{e}\left(h^{m+1}+\Delta t\right), \\
\left\|v-v_{h}\right\|_{l^{2}\left(0, t_{n} ; \mathbf{H}^{1}(D)\right)} \leq c_{c}\left(h^{m}+\Delta t\right) .
\end{gathered}
$$

Recently, Galán del Sastre and Bermejo (2011) have implemented the LGBDF2 method as the rotational incremental velocity-correction scheme, see [15], combined with hp finite elements that use modal basis functions. The formulation of this implementation is as follows.

Let $v_{h}^{0}$ given, choose $v_{h}^{1}$ to be a good approximation to $v^{1}$, then for $n \geq 1$ calculate $\left(v_{h}^{n+1}, p_{h}^{n+1}\right) \in \mathbf{X}_{h} \times M_{h}$ through the following steps:

(1) Set $v_{h e}^{n+1}=2 v_{h}^{n}-v_{h}^{n-1}$, then

(2) calculate $p_{h}^{n+1}$ by solving the equation

$$
\begin{gathered}
\left(\nabla p_{h}^{n+1}, \nabla q_{h}\right)=-\frac{1}{2 \Delta t}\left(3 v_{h e}^{n+1}-4 v_{h}^{n} \circ X_{h}^{n, n+1}+v_{h}^{n-1} \circ X_{h}^{n-1, n+1}\right) \\
-\nu\left(\nabla \times \nabla \times v_{h e}^{n+1}, \nabla q_{h}\right)+\left(f^{n+1}, \nabla q_{h}\right) \quad \forall q_{h} \in M_{h}
\end{gathered}
$$

(3) calculate $v_{h}^{n+1}$ by solving the system

$$
\begin{gathered}
\frac{3}{2}\left(v_{h}^{n+1}, u_{h}\right)+\nu \Delta t\left(\nabla v_{h}^{n+1}, \nabla u_{h}\right)=\left(2 v_{h}^{n} \circ X_{h}^{n, n+1}-\frac{1}{2} v_{h}^{n-1} \circ X_{h}^{n-1, n+1}\right) \\
+\Delta t\left(-\nabla p_{h}^{n+1}+f^{n+1}, u_{h}\right) \quad \forall u_{h} \in \mathbf{X}_{h} .
\end{gathered}
$$

In this paper, the authors also study, via numerical examples, the efficiency and accuracy of LG methods versus SL methods when both methods are combined with quadrilateral spectral/hp elements. The conclusions with respect to these issues are: (1) for the same degree $p$ of the polynomials defining the finite element spaces, both LG and SL methods have the same order of asymptotic convergence, however, LG methods show to be more accurate than SL methods for any $p$; (2) concerning the CPU time, LG methods are more efficient than SL methods when $p$ is large, say, $p \geq 4$; this conclusion may depend on the problem.

A different, although equivalent, form of implementing the velocitycorrection -projection BDF2 scheme is given in Xiu and Karniadakis (2001) where they also use spectral/hp elements, but the material derivative is calculated by interpolation (semi-Lagrangian method) instead of Galerkin 


\section{LG methods for Navier-Stokes Equations}

projection as in LG methods. This implementation is as follows:

Let $v_{h}^{0}$ given, choose $v_{h}^{1}$ to be a good approximation to $v^{1}$, then for $n \geq 1$ calculate $\left(v_{h}^{n+1}, p_{h}^{n+1}\right) \in \mathbf{X}_{h} \times M_{h}$ through the following steps:

(1) Set $\widehat{v}_{h}=2 \bar{v}_{h}^{n}-\frac{1}{2} \bar{v}_{h}^{n-1}$, where $\bar{v}_{h}^{n}$ and $\bar{v}_{h}^{n-1}$ denote the values of $v_{h}^{n}$ and $v_{h}^{n-1}$ at the feet $X_{h}^{n, n+1}\left(x_{i}\right)$ and $X_{h}^{n-1, n+1}\left(x_{i}\right)$ respectively, $x_{i}$ being mesh points; these values are calculated by polynomial interpolation instead of Galerkin projection.

(2) Calculate $p_{h}^{n+1}$ by solving the equation

$$
\left(\nabla p_{h}^{n+1}, \nabla q_{h}\right)=\frac{-1}{\Delta t}\left(\operatorname{div} \widehat{v}_{h}, q_{h}\right)+\left(\frac{\partial p_{h}^{n+1}}{\partial \mathbf{n}}, q_{h}\right), \quad \forall q_{h} \in M_{h} .
$$

(3) Set

$$
\widehat{\widehat{v}}_{h}=\widehat{v}_{h}-\Delta t p_{h}^{n+1}
$$

and calculate $v_{h}^{n+1}$ by solving the system

$$
\begin{array}{r}
\frac{3}{2}\left(v_{h}^{n+1}, u_{h}\right)+\Delta t \nu\left(\nabla v_{h}^{n+1}, \nabla u_{h}\right)=\left(\widehat{\widehat{v}}_{h}, u_{h}\right) \\
+\Delta t\left(f^{n+1}, u_{h}\right), \quad \forall u_{h} \in \mathbf{X}_{h},
\end{array}
$$

where

$$
\frac{\partial p_{h}^{n+1}}{\partial \mathbf{n}}=-\nu \mathbf{n} \cdot\left(\widehat{v}_{h}+\nabla \times\left(\nabla \times v_{h}^{n+1}\right)\right)
$$

\section{LG methods for very high Reynolds numbers}

In general, the LG methods presented so far have serious difficulties in dealing with flows at high Reynolds numbers, unless the mesh is very fine and the quadrature rules to evaluate the integrals (15) are very accurate. As in Eulerian methods, one technique to make LG methods suitable for such Reynolds numbers is to add stabilizing terms to the LG formulation of the Navier-Stokes equations. Bermejo and Saavedra (2015) have introduced a local projection stabilized formulation of LG methods that stabilizes the LG formulation symmetrically, maintaining the Stokes problem structure of the conventional LG formulation; moreover, the local projection stabilization approach can be identified with a variational multi-scale method. This new stabilized LG method is valid for Taylor-Hood finite element spaces $\left(\mathbf{X}_{h}, M_{h}\right)$ so that $m_{1}=m-1$, with $m \geq 2$ in two-dimensional problems and $m \geq 3$ in three-dimensional problems, and the generalized min-element 


\section{R. Bermejo, L. Saavedra}

with $m \geq 1$ in two- or three-dimensional problems. A good reference for the local projection stabilization technique is the textbook [24].

The formulation of the stabilized LG-BDF2 method of [3] reads as follows:

for $j=1,2, \ldots, N-1$, find $\left(v_{h}^{j+1}, p_{h}^{j+1}\right) \in \mathbf{X}_{h} \times M_{h}$ such that for any $v_{h} \in \mathbf{X}_{h}$ and $q_{h} \in M_{h}$ they are solution of the discrete problem

$$
\left\{\begin{array}{l}
\frac{1}{2 \Delta t}\left(3 v_{h}^{j+1}-4 v_{h}^{j} \circ X_{h}^{j, j+1}+v_{h}^{j-1} \circ X_{h}^{j-1, j+1}, u_{h}\right) \\
+\nu\left(\nabla v_{h}^{j+1}, \nabla u_{h}\right)-\left(p_{h}^{j+1}, \operatorname{div} u_{h}\right)+S_{h}\left(v_{h}^{j+1}, u_{h}\right)=\left(f^{j+1}, u_{h}\right), \\
\left(\operatorname{div} v_{h}^{j+1}, q_{h}\right)=0,
\end{array}\right.
$$

here, $S_{h}\left(v_{h}, v_{h}\right)$ is the stabilization term given by the expression

$$
S_{h}\left(v_{h}, u_{h}\right)=\sum_{K \in \Omega_{h}} \tau_{K}\left(\kappa_{h}^{1} \nabla v_{h}, \kappa_{h}^{1} \nabla u_{h}\right)_{K}+\mu_{K}\left(\text { div } v_{h}, \text { div } u_{h}\right)_{K},
$$

where $K$ denotes a generic element of the mesh, and $\tau_{K}$ and $\mu_{K}$ are coefficients that depend on the mesh size and their optimal values are determined by the error analysis; specifically, in the examples we show below, $\tau_{K}=c_{1} h_{K}^{2}, \mu_{K}=c_{2}, c_{1}$ and $c_{2}$ are constants. Moreover, $\kappa_{h}^{1}=\mathbf{i d}-\pi_{h}^{1}$ is the so called fluctuation operator, with id : $\mathbf{L}^{2}(\Omega) \rightarrow \mathbf{L}^{2}(\Omega)$ being the identity operator and $\pi_{h}^{1}: \mathbf{L}^{2}(\Omega) \rightarrow \mathbf{G}_{h}^{1}$ being an orthogonal projector; the finite dimensional space $\mathbf{G}_{h}^{1}$ is defined as

$$
\mathbf{G}_{h}^{1}:=\left\{u_{h} \in \mathbf{L}^{2}(\Omega):\left.u_{h}\right|_{K} \in \mathbf{P}_{0}(K)\right\},
$$

where $\mathbf{P}_{0}(K)$ denotes the space of polynomials of degree zero defined in $K$. In [3] it is proved the following result.

Theorem 7.1. Under the hypotheses of Theorem 1 and with $\tau_{K}=$ $O\left(h_{K}^{2(m-1)}\right)$ there are constants $C_{1}$, which is independent of $h$ and $\Delta t$, but depending on $\|\nabla v\|_{L^{\infty}\left(L^{\infty}(\Omega)\right)}$, and $C_{2}$ independent of $h$ and $\Delta t$, such that

$$
\left\|\mid v^{N}-v_{h}^{N}\right\| \|_{\nu} \leq C_{1}\left(h^{m}+\Delta t^{2}\right)
$$

and

$$
\left\|p-p_{h}\right\|_{l^{2}\left(L^{2}(\Omega)\right)} \leq C_{2}\left(\left\|v-v_{h}\right\|_{l^{\infty}\left(\mathbf{L}^{2}(\Omega)\right)}+h^{m}+\Delta t^{2}\right),
$$

where $\||\cdot|\|_{\nu}$ is a mesh dependent norm given by the expression

$$
\left\|u^{N}\right\|_{\nu}=\left(\left\|u^{n}\right\|_{\mathbf{L}^{2}(\Omega)}+\nu \Delta t \sum_{j=0}^{N}\left\|\nabla u^{j}\right\|_{\mathbf{L}^{2}(\Omega)}+\Delta t \sum_{j=0}^{N} S_{h}\left(u^{j}, u^{j}\right)\right)^{1 / 2} .
$$




\section{LG methods for Navier-Stokes Equations}

\subsection{Numerical test with stabilized $L G$ methods}

To illustrate the performance of the stabilized LG methods, we show some simulations of the flow past the NACA0012 airfoil at zero angle of attack for $\mathrm{Re}=10^{5}$ using the stabilized LG-BDF1 method with $\mathbb{P}_{2} / \mathbb{P}_{1}$ elements and $\Delta t=10^{-3}$. The domain $\Omega:=(-5,10) \times(-5,5)$ and the NACA0012 profile is defined in $0 \leq x \leq 1$. The triangular mesh has 71785 elements of different size with 182893 velocity nodes and 37036 pressure nodes. A detail of the mesh is displayed in Figure 9.
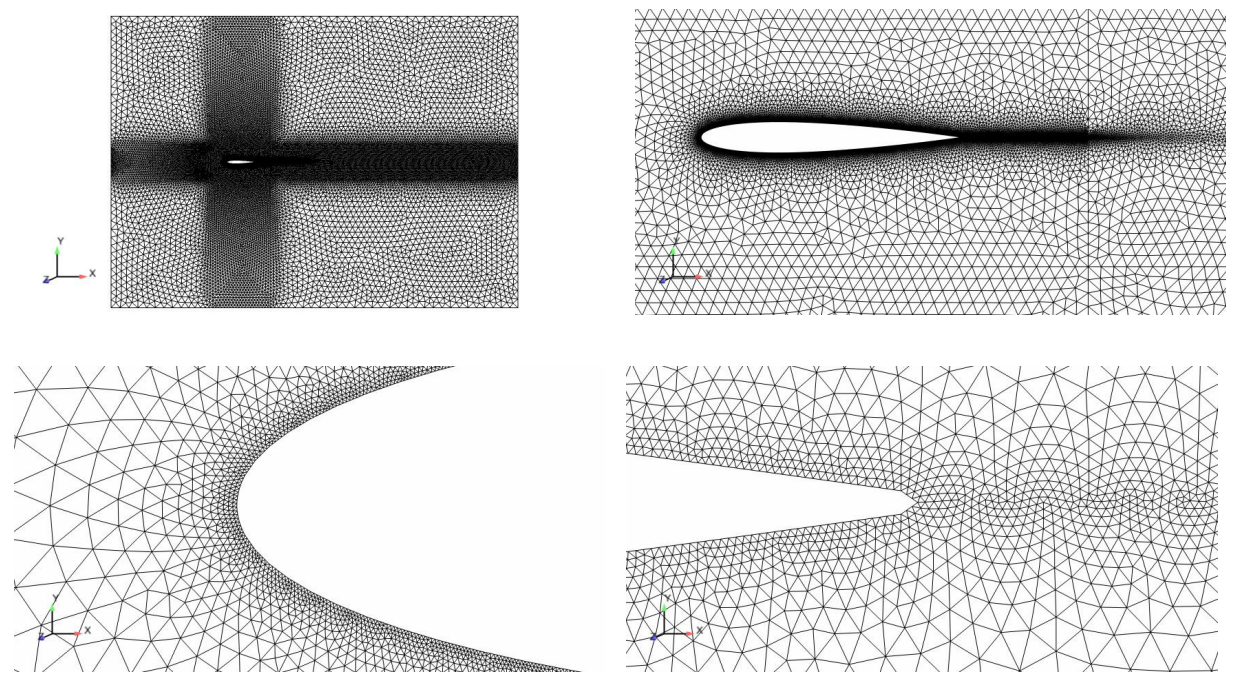

Figure 9. Mesh of the whole domain and around the airfoil (top), and near the leading and trailing edges (bottom)

Since in two dimensional flows the width of the boundary layer developed around the foil is $R e^{-1 / 2}$, then we choose a mesh size $h=10^{-3}$ in a region around the foil to properly resolve the boundary layer. The boundary conditions are the following: (1) no-slip on the foil, (2) a potential velocity $\mathbf{U}_{\infty}=(1,0)$ on the boundary $\{x=-5,-5 \leq y \leq 5\} \cup\{-5 \leq x \leq 10, y=$ $\pm 5\}$, whereas a do-nothing boundary condition is enforced on the outflow boundary. The initial condition is zero in $\Omega \cup\{x=5,-5<y<5\}$, and $\mathbf{U}_{\infty}$ on $\{x=5,-5 \leq y \leq 5\} \cup\{-5 \leq x \leq 5, y= \pm 5\}$. We show in Figure 10 velocity contours of the solutions obtained with both the conventional LG method and the stabilized LG method, the latter with $\tau_{K}=0.1 h_{K}^{2}$ and $\tau_{K}=h_{K}^{2}$, at $t=5 \mathrm{~s}$. A simple inspection of the graphics reveals that the solution of the conventional LG method is unstable, in contrast with the solutions of the local projection stabilized LG method which are stable; as expected, the solution with $\tau_{K}=h_{K}^{2}$ is smoother than the one with $\tau_{K}=0.1 h_{K}^{2}$. Further results with a Reynolds number as high as $R e=3 \times 10^{6}$ are reported in [3]. 


\section{R. Bermejo, L. Saavedra}

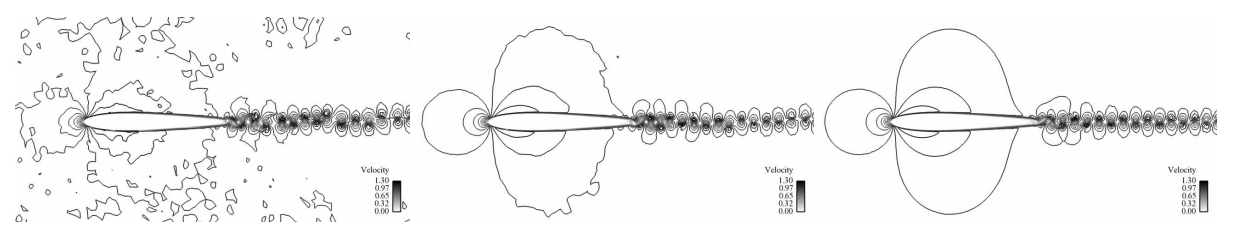

Figure 10. Velocity contours at $t=5 \mathrm{~s}$ obtained with conventional LG method (left) and with stabilized LG method: $\tau_{K}=0.1 h_{K}^{2}$ (middle) and $\tau_{K}=h_{K}^{2}$ (right)

\section{REFERENCES}

1. Y. Achdou, J.-L.Guermond, Convergence analysis of a finite element projection/Lagrange-Galerkin method for the incompressible NavierStokes equations, SIAM Journal on Numerical Analysis, vol. 37, pp. 799-826, 2000.

2. A. Allievi, R. Bermejo, A generalized particle search-locate algorithm for arbitrary grids, Journal of Computational Physics, vol. 132, pp.157166, 1997.

3. R. Bermejo, L. Saavedra, A second order in time local projection stabilized Lagrange-Galerkin method for Navier-Stokes equations at high Reynolds numbers, Computers and Mathematics with Applications, to appear, 2015.

4. R. Bermejo, L. Saavedra, Modified Lagrange-Galerkin methods to integrate time dependent incompressible Navier-Stokes equations, SIAM Journal on Scietific Computing, to appear, 2015.

5. R. Bermejo, P. Galán del Sastre and L. Saavedra, A second order in time modified Lagrange-Galerkin finite element method for the incompressible Navier-Stokes equations, SIAM Journal on Numerical Analysis, vol.50, pp. 3084-3109, 2012.

6. R. Bermejo and L. Saavedra, Modified Lagrange-Galerkin methods of first and second order in time for convection-diffusion problems, $\mathrm{Nu}$ merische Mathematik, vol. 120, pp. 601-638, 2012.

7. K. Boukir, Y. Maday, B. Métivet and E. Razanfindrakoto, A high-order characteristics/finite element method for the incompressible NavierStokes equations, International Journal on Numerical Methods in Flu$i d s$, vol. 25, pp. 1421-1454, 1997.

8. M. Braack and T. Richter, Solutions of 3D Navier-Stokes benchmark problems with adaptive finite elements, Computers and Fluids, vol. 35, pp. 372-392, 2006.

9. G.C. Buscaglia, A. Dari, Implementation of the Lagrange-Galerkin method for the incompressible Navier-Stokes equations, International Journal of Numerical Methods in Fluids, vol. 15, pp. 23-36, 1992

10. J. Douglas and T.F. Russell, Numerical methods for convection- 


\section{LG methods for Navier-Stokes Equations}

dominated diffusion problems based on combining the method of characteristics with finite element or finite difference procedures, SIAM Journal on Numerical Analysis, vol. 19, pp. 871-885, 1982.

11. M. El-Amrani, M. Seaid, An $L^{2}$-projection for the GalerkinCharacteristic solution for incompressible flows, SIAM Journal on Scientific Computing, vol. 33, pp. 3110-3131, 2011.

12. P. Galán del Sastre, R. Bermejo, A comparison of semi-Lagrangian and Lagrange-Galerkin hp-FEM methods in convection-diffusion problems, Communications in Computational Physics, vol. 9, pp. 1020-1039, 2011.

13. J.-L. Guermond, P. Minev, Analysis of a projection/characteristic scheme for incompressible flow, Communications in Numerical Methods in Engineering, vol. 19, pp. 535-550, 2003.

14. J.-L. Guermond, P.Minev, J. Shen, Error analysis of pressure-correction schemes for the Navier-Stokes equations with open boundary conditions, SIAM Journal on Numerical Analysis, vol. 43, pp. 239-258, 2005.

15. J.-L. Guermond, J. Shen, On the error estimates for the rotational pressure-correction projection methods, Mathematics of Computation, vol 73 (248), pp. 1719-1737, 2004.

16. E.Hachem, B. Rivaux, T. Kloczko, H. Digonnet and T. Coupez. Stabilized finite element method for incompressible flows with high Reynolds number, Journal of Computational Physics, vol. 229, pp. 8643-8665, 2010.

17. V. John. Higher order finite element methods and multigrid solvers in a benchmark problem for the 3D Navier-Stokes equations, International Journal of Numerical Methods in Fluids, vol. 40, pp. 775-798, 2002.

18. H. C. Ku, R. S. Hirsh and T. D. Taylor. A pseudospectral method for solution of the three-dimensional incompressible Navier-Stokes equations, Journal of Computational. Physics, vol. 70, pp. 439-462, 1987.

19. P.D. Minev, C.R. Ethier, A characteristic/finite element algorithm for the 3D Navier-Stokes equations using unstructured grids, Computer Methods in Applied Mechanics and Engineering, vol. 178, pp. 39-50, 1999.

20. K. W. Morton, A. Priestley and E. Süli, Stability of the LagrangeGalerkin method with non-exact integration, M2AN Mathematical Modelling and Numerical Analysis, vol. 22, pp. 625-653, 1988.

21. H. Notsu and M. Tabata, A single-step characteristic-curve finite element scheme of second order in time for the incompresible Navier-Stokes equations, Journal of Scientific Computing, vol. 38, pp. 1-14, 2009.

22. A. Priestley, Exact projections and the Lagrange-Galerkin method: a realistic alternative to quadrature, Journal of Computational Physics, vol. 112, pp. 316-333, 1994. 


\section{R. Bermejo, L. Saavedra}

23. O. Pironneau, On the transport-diffusion algorithm and its applications to the Navier-Stokes equations, Numerische Mathematik, vol. 38, pp. 309-332, 1982.

24. H.-G. Roos, M. Stynes, Robuts Numerical Methods for Singularly Perturbed Differential Equations, Springer Series in Computational Mathematics, Springer-Verlag, Berlin, 2008.

25. M. Schäfer and S. Turek, Benchmark computations of laminar flow around a cylinder (With support by Durst F, Krause E, Rannacher R). In: Schäfer E, editor. Flow simulation with high-performance computers II. DFG priority research program results 1993-1995. Notes numer. fluid mech, no. 52. Wiesbaden Vieweg; 547-566, 1996.

26. C. Shu, L. Wang and Y. T. Chew Numerical computation of threedimensional incompressible Navier-Stokes equations in primitive variable form by DQ method, Intermational Journal for Numerical Methods in Fluids, vol.43, pp. 345-368, 2003.

27. E. Süli, Convergence and nonlinear stability of the Lagrange-Galerkin method for the Navier-Stokes equations, Numerische Mathematik, vol.54, pp. 459-483, 1988.

28. L.Q. Tang, T. Cheng and T.T.H. Tsang Transient solutions for threedimensional lid-driven cavity flows by a least-squares finite element method, Intermational Journal for Numerical Methods in Fluids, vol. 21, pp. 413-432, 1995.

29. C. Temperton, A. Staniforth, An efficient two-time-level semiLagrangian semi-implicit integration scheme, Quaterly Journal of the Royal Meteorological Society, vol. 113, pp. 1025-1039, 1987.

30. D.Xiu and G.E.Karniadakis, A semi-Lagrangian high order method for Navier-Stokes equations, Journal of Computational Physics, vol.172, pp. 658-684, 2001. 